Mots. Les langages du politique

102 | 2013

Les discours sur l'enseignement supérieur et la recherche

La terminologie de l'enseignement supérieur en Belgique francophone transposée dans le système d'intégration européenne. Raisons et conséquences d'une harmonisation linguistique manquée

Nathalie Lemaire et Marc van Campenhoudt

\title{
CpenEdition
}

Journals

Édition électronique

URL : http://journals.openedition.org/mots/21314

DOI : $10.4000 /$ mots. 21314

ISSN : 1960-6001

Éditeur

ENS Éditions

Édition imprimée

Date de publication : 2 septembre 2013

ISBN : 978-2-84788-414-2

ISSN : 0243-6450

Référence électronique

Nathalie Lemaire et Marc van Campenhoudt, « La terminologie de l'enseignement supérieur en

Belgique francophone transposée dans le système d'intégration européenne. Raisons et

conséquences d'une harmonisation linguistique manquée ", Mots. Les langages du politique [En ligne], 102 | 2013, mis en ligne le 02 septembre 2015, consulté le 30 avril 2019. URL : http:// journals.openedition.org/mots/21314; DOI : 10.4000/mots.21314 


\section{La terminologie de l'enseignement supérieur en Belgique francophone transposée dans le système d'intégration européenne. Raisons et conséquences d'une harmonisation linguistique manquée}

Dans le cadre de l'harmonisation des cursus de l'enseignement supérieur de l'Union européenne, les États membres disposaient de toute latitude pour revoir leur terminologie du domaine ${ }^{1}$.

En France, la nouvelle nomenclature licence-master-doctorat $(L M D)^{2}$ semble s'être implantée dans l'usage sans entrainer de confusion ni de difficultés langagières majeures. Il est vrai qu'à la différence de la Belgique francophone, la France disposait déjà d'une terminologie pour désigner les diplômes obtenus au terme de trois, cinq ou huit années d'études. En Belgique francophone, l'autorité compétente 3 a transposé, en 2004 , les grades universitaires sanctionnant les trois cycles d'études supérieures définis par le processus d'intégration dans un décret, dit décret de Bologne (Moniteur belge, 2004), sans concertation avec les institutions francophones responsables de la politique linguistique4. La nomenclature de désignation des grades académiques fixée par ce décret de référence recèle des anomalies et incohérences linguistiques

1. Déclaration de Bologne du 19 juin 1999 - Déclaration conjointe des ministres européens de l’Éducation.

2. Nous appliquons les conventions graphiques suivantes : italique pour les emplois autonymes, les titres de publications, les termes ou locutions d'origine étrangère non lexicalisés, la mise en évidence d'éléments notionnels; chevrons «〉 pour les notions; chevron > pour la dérivation ; guillemets français pour les citations; guillemets anglais pour les traits grammaticaux.

3. Le ministre de l’Enseignement supérieur de la Fédération Wallonie-Bruxelles (FWB).

4. Nommément, le Service de la langue française et le Conseil de la langue et de la politique linguistique de la FWB.

Centre de recherche en linguistique appliquée TERMISTI

Institut supérieur de traducteurs et interprètes - Haute École de Bruxelles nlemaire@termisti.org

Centre de recherche en linguistique appliquée TERMISTI

Institut supérieur de traducteurs et interprètes - Haute École de Bruxelles mvc@termisti.org 
de divers ordres, qui nuisent à la lisibilité des documents émanant de l'autorité publique et qui entravent la communication avec et entre les usagers - lesquels développent depuis plus de six ans des stratégies d'aménagement non coordonnées.

Pour bien faire comprendre les difficultés rencontrées par les locuteurs belges francophones lorsqu'ils sont amenés à parler ou à écrire au sujet de leurs cursus ou diplômes universitaires, nous nous proposons de commencer par présenter la terminologie fixée par le décret de Bologne et d'en exposer les failles. Nous tâcherons ensuite de cerner, à titre de conclusion, l'enjeu sociétal que révèle la persistance de cette situation problématique.

Cette contribution est nourrie par notre travail régulier de recherches en terminologie commanditées par le Service de la langue française (SLF) de la Fédération Wallonie-Bruxelles (FWB). S'agissant d'alimenter la base de données BelTerme5, elles inspirent les avis que le Conseil de la langue française et de la politique linguistique fournit au pouvoir exécutif, mais n'exercent aucune influence sur les choix lexicaux du législateur. Par ailleurs, la méthodologie adopte une perspective strictement monosémique, non par point de vue doctrinal, mais pour répondre aux exigences de l'ingénierie linguistique et de la gestion de l'équivalence dans un cadre multilingue ${ }^{6}$.

\section{Problèmes linguistiques posés par la nouvelle nomenclature}

Notre attention se fixera sur les dénominations des grades de premier et deuxième cycles, toutes deux forgées pour les besoins de la réforme de l'enseignement supérieur européen. Le grade de troisième cycle, docteur, qui ne pose aucun problème d'ordre morphosyntaxique et qui a pu être intégré tel quel dans la nouvelle nomenclature, nous servira de paradigme.

\section{Comparaison ante/post de la terminologie fixée parles sources légales}

L'ancienne architecture des études supérieures se différenciait en Belgique par l'absence de diplôme universitaire au niveau bac + 3. L'étudiant effectuait un premier cycle en deux ans (candidature) et un second cycle en deux ans (licence). Comme en France, l'étudiant pouvait effectuer une cinquième année de spécialisation à la recherche (DEA) ou dans une voie professionnelle (DESS). Hors université, on suivait des études professionnalisantes en trois ans, nommées graduat ou régendat7.

5. En ligne: http://www2.cfwb.be/franca/xml/html/bd/bd.htm (dernière consultation : 27 mars 2013).

6. Les fiches rédigées en français proposent des équivalences en allemand et en néerlandais.

7. Pour les futurs enseignants du secondaire inférieur. 
La réforme a conduit à distinguer un cycle de bachelier (universitaire ou professionnalisant), un cycle de master et un cycle de doctorat. Les tableaux $1 a$ et $1 b$ présentent l'ancienne et la nouvelle architecture.

\begin{tabular}{|c|c|c|c|c|c|}
\hline \multicolumn{3}{|c|}{ 1a: Avant le décret de Bologne } & \multicolumn{3}{|c|}{$1 \mathrm{~b}:$ Après le décret de Bologne } \\
\hline 8 & \multirow{3}{*}{ doctorat } & & 8 & \multirow{3}{*}{ doctorat } & \\
\hline 7 & & & 7 & & \\
\hline 6 & & & 6 & & \\
\hline 5 & DEA/DESS* & & 5 & \multirow{2}{*}{ master } & \\
\hline 4 & \multirow{2}{*}{ licence } & & 4 & & \\
\hline 3 & & \multirow{3}{*}{ graduat/régendat } & 3 & \multirow{3}{*}{$\begin{array}{l}\text { bachelier de } \\
\text { transition }\end{array}$} & \multirow{3}{*}{$\begin{array}{c}\text { bachelier } \\
\text { professionnalisant }\end{array}$} \\
\hline 2 & \multirow[t]{2}{*}{ candidature } & & 2 & & \\
\hline 1 & & & 1 & & \\
\hline & Type long & Type court & & Type long & Type court \\
\hline
\end{tabular}

*La schématisation proposée est nécessairement réductrice par rapport à la diversité des curriculums. Outre le DEA et le DESS, il conviendrait, par exemple, de mentionner ici les cas de licences en 5 ans (droit, pharmacie, dentisterie...).

\section{Tableaux 1a et $1 \mathrm{~b}$ : Architecture des études avant et après l'adoption du processus de Bologne par la Fédération Wallonie-Bruxelles}

Cette nouvelle architecture, somme toute très aisée à appréhender, a semblé poser bien davantage de problèmes de dénomination aux juristes chargés de désigner tantôt le grade universitaire, tantôt le titulaire de ce grade, tantôt le cursus donnant accès au grade et tantôt encore, l'étudiant suivant ledit cursus.

Le tableau 2 présente la terminologie officielle des grades (et notions essentielles associées) de l'enseignement supérieur en Belgique francophone (désormais Fédération Wallonie-Bruxelles ou FWB) avant et après le processus d'intégration européenne.

À la lecture du tableau 2, page suivante, il apparait que la terminologie extraite du décret de Bologne du gouvernement de Fédération WallonieBruxelles (FWB) et des textes juridiques qui en découlent se caractérise par deux aspects. Tout d'abord, par ses lacunes linguistiques; ensuite, par l'asymétrie des procédés qui ont présidé à la création néologique des nouveaux grades. Penchons-nous ici sur ces deux caractéristiques.

\section{Vides notionnels dans la famille des termes désignant les nouveaux grades académiques}

Seuls les nouveaux grades académiques, bachelier et master, sont explicitement désignés et définis par le législateur. Contrairement à ce que l'on observait dans la terminologie de l'ancien système d'enseignement supérieur 


\begin{tabular}{|c|c|c|c|c|c|}
\hline & & $\langle$ grade〉 & $\begin{array}{l}\text { 〈titulaire } \\
\text { du grade〉 }\end{array}$ & $\begin{array}{l}\text { 〈cursus donnant } \\
\text { accès } \\
\text { au grade» }\end{array}$ & 〈étudiant〉 \\
\hline \multirow{3}{*}{$\begin{array}{l}\text { Avant } \\
2004\end{array}$} & cycle 1 & $\begin{array}{l}\text { régent-e/ } \\
\text { gradué-e/ } \\
\text { candidat-e* }\end{array}$ & $\begin{array}{l}\text { régent-e/ } \\
\text { gradué-e/ } \\
\text { candidat-e }\end{array}$ & $\begin{array}{l}\text { régendat / } \\
\text { graduat / } \\
\text { candidature }\end{array}$ & $\begin{array}{l}\text { étudiant en } 1^{\mathrm{re} / 2^{\mathrm{e}} /} \\
\left(3^{\mathrm{e}}\right) \text { année de } \\
\text { régendat / graduat / } \\
\text { candidature }\end{array}$ \\
\hline & cycle 2 & licencié-e & licencié-e & licence & $\begin{array}{l}\text { étudiant en } 1^{\mathrm{re}} / 2^{\mathrm{e}} \\
\text { année de licence }\end{array}$ \\
\hline & cycle 3 & docteur-e & docteur-e & doctorat & doctorant-e \\
\hline \multirow{3}{*}{$\begin{array}{l}\text { Selon } \\
\text { le décret } \\
\text { de } \\
\text { Bologne } \\
(2004)\end{array}$} & cycle 1 & 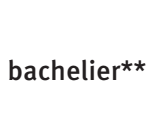 & $\begin{array}{l}\text { titulaire d'un } \\
\text { diplôme de } \\
\text { bachelier }\end{array}$ & $\begin{array}{l}\text { études de } \\
\text { bachelier }\end{array}$ & $\begin{array}{l}\text { étudiant inscrit en } \\
1^{\text {re }} / 2^{\mathrm{e}} / 3^{\mathrm{e}} \text { année de } \\
\text { programme de } \\
\text { bachelier }\end{array}$ \\
\hline & cycle 2 & master** & $\begin{array}{l}\text { titulaire d'un } \\
\text { diplôme de } \\
\text { master }\end{array}$ & $\begin{array}{l}\text { études de } \\
\text { master }\end{array}$ & $\begin{array}{l}\text { étudiant inscrit en } \\
1^{r e} / 2^{e^{\star \star \star}} \text { année } \\
\text { de programme de } \\
\text { master }\end{array}$ \\
\hline & cycle 3 & docteur-e & docteur-e & doctorat & doctorant-e \\
\hline
\end{tabular}

* Les concepts explicitement définis par les textes de loi sont présentés en gras.

** Dans le texte du décret, on lit Bachelier et Master avec une majuscule abusive à l'initiale. Nous faisons le choix de neutraliser la variation de casse dans la suite de notre argumentation de manière à ne pas amalgamer des problèmes relevant de niveaux différents.

*** Notons que les masters de médecine vétérinaire comptent 180 crédits ( 3 ans) et ceux de médecine 240 ( 4 ans).

Tableau 2. Terminologie des grades et notions associées de l'enseignement supérieur en Belgique francophone tirée des textes de loi avant et après le décret d'harmonisation européenne (2004)

en FWB ${ }^{8}$ et contrairement aux options terminologiques retenues en France9, les curriculums ou programmes de formation afférents aux grades intégrés à l'espace européen ne sont désignés qu'au moyen de périphrases : études de bachelier et études de master. L'absence de dénomination propre désignant les programmes de cours est, nous semble-t-il, à l'origine de la difficulté fondamentale à laquelle ont été - et sont, du reste, toujours - confrontés les experts du domaine en situation de communication vers le public.

Le recours à la périphrase matricielle études de bachelier/études de master devait immanquablement entrainer, en cascade, des périphrases toujours

8. Où l'on disposait des couples dérivés suivants : licence >licencié-e, candidature >candidat-e, régendat > régent-e, graduat > gradué-e.

9. Où, avec le système $L M D$, la terminologie officielle prévoit les dénominations des programmes de formation. 
plus longues et d'autant moins commodes en situation de communication pratique. Si l'on prend comme point de référence le paradigme de la famille du terme docteur, rescapé de l'ancienne nomenclature, et que l'on s'en tient strictement à la terminologie présentée dans le décret de Bologne, aux termes doctorant et docteur en répondent, respectivement, dans toute leur longueur, les fastidieuses tournures étudiant inscrit à un programme de bachelier/master en et titulaire / détenteur / diplômé d'un diplôme/titre / grade de bachelier/master en $\sim 10$.

Nous verrons plus bas que l'impraticabilité de ces expressions a placé les milieux administratifs (ministère de l'Enseignement supérieur aussi bien qu'institutions d'enseignement supérieur) dans l'obligation de s'écarter quelque peu de cette norme pour mettre en place, tant bien que mal, un usage administratif malmenant moins le principe, bien connu, d'économie linguistique. On touche ici du doigt une problématique récurrente : la terminologie d'un domaine est un instrument de communication au quotidien qui ne peut être pensé uniquement sous l'angle de l'efficacité juridique, sans considération pour l'exactitude et la clarté linguistiques.

\section{Procédés de création néologique asymétriques}

La transposition des objectifs de la déclaration de Bologne dans la loi communautaire de la FWB a été fortement marquée par le système d'enseignement supérieur anglo-saxon. Personne ne s'étonnera de cette influence de l'anglais dans la terminologie de l'enseignement supérieur en FWB, tant les Belges francophones, du fait, vraisemblablement, de leur contexte national plurilingue, semblent plus enclins à intégrer des termes anglais dans leur vocabulaire que leurs voisins outre-Quiévrain ${ }^{11}$ (Dierickx, 1997, p. 313-315).

Ainsi qu'en a disposé le législateur dans le décret de Bologne, en écho au bachelor's degree anglais, la dénomination de l'ancien grade de premier cycle, candidat-e, s'efface au profit du terme bachelier ${ }^{12}$, et le grade de deuxième cycle (anciennement licencié-e), est désormais connu sous la dénomination master, fidèle réplique du master's degree des anglophones.

10. Il convient toutefois de préciser ici que, dans le système d'enseignement supérieur antérieur à la réforme de Bologne, seul le terme docteur disposait d'une dénomination propre pour désigner la notion d'〈étudiant inscrit à un programme de formation〉. Les termes désignant les autres grades académiques candidat-e, licencié-e, gradué-e, régent-e n'offraient pas cette possibilité et, dans ce seul cas, le recours à une périphrase du type étudiant en ixième candidature / licence/... s'imposait, là aussi.

11. En dépit de leur notoire résistance à l'anglicisation de leur langue, les Français n'ont, du reste, eux-mêmes pas résisté complètement à la prédominance de l'anglais dans l'Union puisque leur schéma européen d'organisation des études, LMD (licence / master / doctorat), comporte, en son milieu, le pur anglicisme master - en concurrence, toutefois, avec la variante francisée mastère.

12. Non féminisé, contrairement aux prescriptions du décret de féminisation des noms de métiers, grades et titres adopté par le Parlement de la FWB le 21 juin 1993. 
Si bachelier et master partagent une inspiration anglo-saxonne, ces dénominations, bien que créées simultanément, résultent en revanche de procédés néologiques distincts.

Le terme bachelier, qui existait déjà dans la langue française, trouve ici une nouvelle acception (‘titulaire d'un grade obtenu au terme d'un premier cycle d'études universitaire`), par ailleurs déjà présente dans les universités québécoises. En tant que terme remotivé, sa prononciation ne pose aucun problème au locuteur francophone et sa structure morphosyntaxique semble a priori lisible grâce à son suffixe -ier, qui apparente le terme à la longue série des agents animés désignés par suffixation : école > écolier, grue > grutier ${ }^{13}$...

Il en va, par contre, tout autrement de master, emprunt quasi direct dont la prononciation demeure hasardeuse en français régional de Belgique - [maste: R] ou [mastœ : R] ou, à l'anglaise, ['ma : stər].

\section{Observation de l'usage administratif postérieur au décret de Bologne : homonymie de 〈grade〉 et de 〈curriculum〉}

Si l'on compare le tableau $3^{14}$, présentant la terminologie extraite du décret de Bologne et des textes juridiques qui en découlent, avec le tableau 4, présentant l'usage administratif observé à partir de 2004, on voit que, pour désigner les curriculums, la périphrase études de bachelier/master a disparu, sous la plume des fonctionnaires, au profit des formes elliptiques bachelier et master.

\begin{tabular}{|c|c|c|c|}
\hline 〈grade〉 & $\langle$ titulaire du grade $\rangle$ & $\begin{array}{l}\text { «curriculum } \\
\text { donnant } \\
\text { accès au grade» }\end{array}$ & $\begin{array}{l}\text { 〈personne inscrite } \\
\text { au programme de formation } \\
\text { donnant accès au grade〉 }\end{array}$ \\
\hline bachelier & $\begin{array}{l}\text { titulaire d'un diplôme } \\
\text { de bachelier }\end{array}$ & $\begin{array}{l}\text { études } \\
\text { de bachelier }\end{array}$ & $\begin{array}{l}\text { étudiant-e inscrit-e } \\
\text { à un programme } \\
\text { de bachelier }\end{array}$ \\
\hline master & $\begin{array}{l}\text { titulaire d'un diplôme } \\
\text { de master }\end{array}$ & $\begin{array}{l}\text { études } \\
\text { de master }\end{array}$ & $\begin{array}{l}\text { étudiant-e inscrit-e } \\
\text { à un programme } \\
\text { de master }\end{array}$ \\
\hline docteur-e & docteur-e & doctorat & doctorant \\
\hline
\end{tabular}

Tableau 3. Description de la terminologie des grades de $1^{\mathrm{er}}, 2^{\mathrm{e}}$ et $3^{\mathrm{e}}$ cycles extraite du décret de Bologne (rappel)

13. Nous verrons plus loin que cette structure morphosyntaxique, correctement identifiée dans l'usage informel intuitif des étudiants, a échappé à l'Administration, puisque l'usage administratif désigne par bachelier le programme de formation et le grade de premier cycle et non le grade et le diplômé (ou l'étudiant) de $1^{\text {er }}$ cycle.

14. Pour la facilité de lecture, nous insérons, plus bas, le tableau 3, qui reprend la partie inférieure du tableau 2. 


\begin{tabular}{|c|c|c|c|}
\hline 〈grade〉 & $\langle$ titulaire du grade $\rangle$ & $\begin{array}{l}\text { «curriculum donnant } \\
\text { accès au grade» }\end{array}$ & $\begin{array}{c}\text { «personne inscrite } \\
\text { au programme } \\
\text { de formation } \\
\text { donnant accès au grade〉 }\end{array}$ \\
\hline bachelier & $\begin{array}{l}\text { titulaire } \\
\text { d'un diplôme } \\
\text { de bachelier }\end{array}$ & bachelier & $\begin{array}{l}\text { étudiant(e) inscrit } \\
\text { à un programme de bache- } \\
\text { lier }\end{array}$ \\
\hline master & $\begin{array}{l}\text { titulaire } \\
\text { d'un diplôme } \\
\text { de master }\end{array}$ & master & $\begin{array}{l}\text { étudiant(e) inscrit } \\
\text { à un programme de master }\end{array}$ \\
\hline docteur-e & docteur-e & doctorat & doctorant \\
\hline
\end{tabular}

Tableau 4. Description de la terminologie des grades de $1^{\mathrm{er}}, 2^{\mathrm{e}}$ et $3^{\mathrm{e}}$ cycles dans l'usage administratif après le décret de Bologne

De ce fait, la traditionnelle identité de dénomination entre le grade et le titulaire du grade (voir docteur en gras et en italique dans le tableau 4) cède la place à un phénomène inédit d'homonymie entre le grade et le curriculum donnant accès au grade (voir bachelier et master en gras dans le tableau 4) ${ }^{15}$.

En effet, en français de référence, dans le domaine de l'enseignement supérieur comme dans celui de la défense, par exemple, (gradè et 〈gradé〉 partagent en principe la même dénomination : un capitaine (〈gradé») est une personne qui porte le 〈grade de capitaine〉, tout comme les gradués, régents, candidats, ou licenciés (〈porteurs de grade〉) étaient, dans l'ancienne nomenclature belge, titulaires du grade de gradué, de régent, de candidat ou de licencié. La terminologie administrative de l'enseignement supérieur réformé rompt donc avec cette tradition en instaurant une homonymie entre le grade et, non pas son titulaire, mais le curriculum conduisant à son obtention : le 〈grade de bachelier/master〉 s'obtient au terme d'un bachelier/master 〈programme〉.

Pour éviter la confusion, la terminologie administrative a maintenu la périphrase titulaire d'un grade de bachelier/master pour désigner les diplômés. On n'écrirait donc pas, en milieu administratif, «il est bachelier / master en $\sim$ », comme on disait dans l'ancienne nomenclature «il est candidat / licencié en », mais « il a un [grade / diplôme de] bachelier / master en ».

On peut s'interroger sur les raisons qui ont conduit l'usage administratif à réduire les expressions légales désignant les curriculums. N'ayant pas été

15. Nous parlons d'homonymie dès lors que notre approche terminologique s'effectue dans un cadre multilingue. En effet, une équivalence s'établit toujours à l'intérieur d'un espace de sens restreint, contraint par la langue qui offre la plus faible extension. Par ailleurs, la nécessité de permettre l'interversion des langues source et cible au sein d'une même fiche terminologique multilingue implique la création de fiches homonymes pour garantir une équivalence stricte (Van Campenhoudt, 2000 [p. 131-138] et 2001). Ceci explique que nous ne considérions pas dans le présent article l'idée d'un lien métonymique entre les acceptions considérées, même si elle permettrait d'envisager une polysémie, toujours plus aisée à appréhender en langue générale et dans un cadre monolingue. 
associés au processus de création néologique, nous ne pouvons que formuler une hypothèse à ce sujet.

La phraséologie de l'enseignement supérieur, dans sa référence à l'ancienne nomenclature belge, était caractérisée par un emploi abondant de constructions syntaxiques du type [verbe transitif + complément d'objet direct] : on faisait un régendat en telle discipline, on achevait sa licence en $\sim$, on entamait un graduat en , etc. Il est donc permis de penser que le plus urgent, dans l'usage administratif, était de trouver une dénomination commode pour désigner les programmes de formation. La réduction par ellipse des syntagmes périphrastiques études de master/bachelier en master / bachelier a, du reste, pu être facilitée par le manque de lisibilité morphosyntaxique du terme emprunté master, comme nous l'évoquions plus haut.

C'est ainsi qu'il était courant d'entendre dans la bouche du locuteur lambda de Belgique ou d'ailleurs, bien avant la réforme de Bologne déjà, qu'Untel était «parti faire un master en à Oxford » $^{16}$. On peut postuler que, dans ce contexte, l'abrègement elliptique de études de master en master s'est imposé presque naturellement sous la plume du législateur. De là découlerait l'abrègement de études de bachelier en bachelier - qui devait, nous le verrons tout de suite, susciter plus de réticences dans l'usage informel ${ }^{17}$ compte tenu de la valeur sémantique de sa structure morphosyntaxique.

On notera toutefois que ce glissement résulte, à la base, d'une méconnaissance de la structure morphosyntaxique des termes anglais master et bachelor, qui représentent des dénominations d'humains et non des dénominations d'objets inanimés ou abstraits. En effet, ainsi qu'il apparait clairement à la lecture du tableau 5, master désigne, en anglais britannique, le titulaire d'un grade de $2^{\mathrm{e}}$ cycle; et lorsque ce terme réfère à un grade ou à un programme, c'est à l'intérieur d'un syntagme comprenant le 's génitif qui indique la détention de quelque chose par quelqu'un : Master's [degree], Master's programme. De même, bachelor désigne, dans la langue anglaise générale, un jeune homme célibataire. Dans le domaine de l'enseignement, il est, lui aussi, en principe, utilisé avec le génitif 's : Bachelor's [degree], Bachelor's [programme].

16. Sentiment linguistique corroboré par une rapide recherche sur Internet à l'aide des outils de

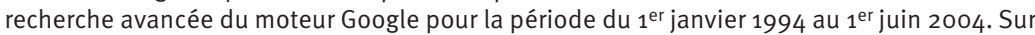
les forums, essentiellement, des internautes produisent des énoncés tels que : «Je suis partie à l'université de Boston poury faire un master en journalisme» [http://terminushongkong.free. $\mathrm{fr} /$ pays/vietnam/pj_15_v_trou.html], dernière consultation le 27 février 2013 ; "Je cherche une Université francophone poury faire un Master en droit des affaires » [http://www.educh.ch/coaching-formation-emploi.php?question=65891], dernière consultation le 27 février 2013.

17. Par informel, nous entendons : libéré de la contrainte du langage officiel. Par exemple, dans les échanges oraux ou écrits entre étudiants, sur les pages intranet d'un cours ou encore dans le procès-verbal d'une réunion de travail. Il s'agit d'une variation diaphasique plutôt que diastratique. 


\begin{tabular}{|c|c|c|c|}
\hline & 〈grade〉 & 〈diplômé〉 & 〈curriculum〉 \\
\hline \multirow{2}{*}{ UK } & $\begin{array}{l}\text { Bachelor's } \\
\text { [degree] }\end{array}$ & Graduate & Bachelor's programme \\
\hline & Master’s [degree] & Master & Master's programme \\
\hline \multirow{2}{*}{ FWB } & $\begin{array}{l}\text { grade } \\
\text { de bachelier }\end{array}$ & titulaire d'un grade de bachelier & Bachelier \\
\hline & grade de master & titulaire d'un grade de master & Master \\
\hline
\end{tabular}

Tableau 5. Système terminologique anglo-saxon et description de l'usage administratif en FWB

\section{Dans l'usage informel des étudiants : un retour intuitif, mais partiel, à l'homonymie de 〈gradè et de 〈diplômé〉}

Si les responsables administratifs des institutions d'enseignement supérieur sont soucieux de respecter les prescriptions de l'usage administratif et s'astreignent ${ }^{18}$ à considérer bachelier comme un programme de formation dans leurs écrits, les étudiants de notre institution de rattachement n’ont, eux, pas intégré cet usage ${ }^{19}$. Comme on le voit dans le tableau 6 ci-dessous, la majorité des étudiants ayant répondu, en 2007 et en 2012, à notre enquête d’implantation reconnaissent intuitivement le sens étymologique et la structure morphosyntaxique qui désignent bachelier comme 〈titulaire d'un grade〉, et, conséquemment, restaurent spontanément sa flexion féminine, bachelière.

Le terme master entendu comme 〈programme de formation s passe, lui, sans aucune difficulté dans l'usage étudiant, car sa motivation morphosyntaxique anglaise de mot présentant le trait grammatical "animé” n’est pas identifiée par les locuteurs francophones.

On se trouve, donc, face à une nouvelle forme de traitement asymétrique des dénominations de grade : le 〈grade de premier cycle〉, bachelier, est homonyme du 〈diplômé de ce grade〉. Tandis que le 〈grade de deuxième cycle〉, master,

18. Interrogés sur le sujet, certains précisent spontanément que cette manière de procéder heurte leur représentation linguistique.

19. Conclusions tirées des résultats d'une enquête d'usage, menée une première fois en 2007, puis une seconde fois, sans modification majeure des tendances observées, en 2012, auprès de nos étudiants de $4^{\mathrm{e}}$ année de l'Institut supérieur de traducteurs et interprètes de la Haute École de Bruxelles. Le questionnaire et le détail des réponses fournies par les étudiants peuvent être demandés aux auteurs. Cette enquête annexe, non prévue par le cahier des charges de l'appel d'offres, et menée de notre propre initiative à des fins de vérification du caractère problématique des nouvelles dénominations de grades et de curriculums en discours semi-libre, n'a, en effet, pas fait l'objet d'une publication. Il serait certainement intéressant d'élargir ce sondage à des étudiants inscrits dans des filières de formation a priori moins sensibles aux enjeux syntaxiques et sémantiques - ce que ne permettait pas l'objectif du projet de recherche, lequel nous avait été commandé dans un cadre qui n'échappait ni aux contraintes de temps ni aux limitations budgétaires. 
mal interprété par rapport à l'anglais auquel il a été emprunté, est quant à lui considéré comme un homonyme du «curriculum donnant accès à ce gradè.

\begin{tabular}{llll}
\hline 〈grade〉* & 〈diplômé〉 & 〈curriculum〉 & $\begin{array}{l}\text { 〈personne inscrite } \\
\text { au curriculum〉 }\end{array}$ \\
\hline (bachelier) & bachelier, -ière & bac[calauréat] & $\begin{array}{l}\text { étudiant(e) en [/de] } \\
\text { énième bac }\end{array}$ \\
\hline (master) & diplômé [d’un master] & master & $\begin{array}{l}\text { étudiant(e) en [/de] } \\
\text { énième master }\end{array}$ \\
\hline
\end{tabular}

*Le questionnaire, centré sur l'observation des stratégies d'évitement ou de contournement des difficultés périphrastiques découlant de la terminologie officielle lacunaire, ne prévoyait pas de question vérifiant la connaissance (supposée connue) de la dénomination des grades par les étudiants.

Tableau 6. Description de la terminologie des grades de premier et deuxième cycles dans l'usage des étudiants en 2007 et en $2012^{20}$

Pour combler le vide dénominationnel laissé par le retour, logique, de bachelier dans la case 〈diplômé〉, c’est le terme baccalauréat - y compris sous sa forme abrégée bac -, évité par l'usage administratif21, qui fait son apparition dans l'usage informel.

Autre fait observable, les périphrases désignant les <personnes inscrites à un curriculum > sont raccourcies de facto : de étudiant-e inscrit-e à un programme de master / bachelier, on passe, par ellipse du composant programme de, à étudiant-e inscrit-e en ixième master/bac[calauréat] ou étudiant-e de ixième master/bac[calauréat].

En bref, la terminologie du système d'enseignement supérieur réformé est caractérisée en Belgique francophone par un écart entre :

- la terminologie asymétrique et lacunaire établie par le législateur,

- l'usage administratif des experts consultés et des sites officiels²2, qui limite le recours à la périphrase en introduisant l'homonymie entre grades et curriculums sans respecter la structure morphosyntaxique des termes master et bachelier,

- et l'usage informel des étudiants qui rétablit, partiellement, l'homonymie - conforme au fonctionnement morphologique de la langue - du grade

20. Réponses majoritaires.

21. De toute évidence pour éviter la confusion avec son homonyme dans le système d'enseignement secondaire en France. À noter que cette homonymie ne pose, par contre, aucun problème au Québec.

22. Ce constat doit toutefois être nuancé. L'usage officiel n'est pas observé en toutes circonstances par les milieux administratifs. On trouve aisément des attestations de baccalauréat utilisé dans le sens de «curriculum du premier cycle〉 sur le site des universités et des hautes écoles dans des pages qui ne relèvent pas de l'administration centrale, mais d'une communication informelle. On le trouvait même jadis sur le site Internet du Cabinet de la ministre de l'Enseignement supérieur Marie-Dominique Simonet : «Les principales modifications dues au décret de Bologne. Deux cycles de bases : le baccalauréat et le master», simonet.wallonie.be, page Université L'enseignement universitaire (consulté le 20 décembre 2007). 
et du porteur de grade et qui comble, en partie, les vides notionnels en introduisant le terme baccalauréat.

Ces variations s'expliquent par le fait que le décret de Bologne n'a pas suffisamment balisé les dénominations découlant de la réforme du système éducatif. Les «flous» qu'il laisse apparaitre en usant de périphrases plutôt qu'en proposant explicitement de nouvelles dénominations pour désigner les curriculums, les diplômés et les étudiants débouchent sur ces variations d'usage, préjudiciables à la communication entre les milieux spécialisés et leur public.

\section{(Dé)raisons de l'inertie politique devant les solutions d’aménagement}

Ces anomalies linguistiques, parmi d'autres ${ }^{23}$, ont été relevées et signalées en 2007 par notre centre de recherche dans le cadre d'un rapport commandité par le Service de la langue française (SLF) de la FWB. Concernant les dénominations des notions dérivées des grades de premier et deuxième cycles, le rapport débouchait sur deux propositions de réaménagement terminologique, l'une, théorique, dans la plus pure tradition francophile normative et inspirée du modèle québécois (tableau 7); l'autre, plus réaliste, avec recours partiel à l'emprunt, suivant un scénario de «compromis à la belge » entre usage prédominant et respect des règles linguistiques élémentaires (tableau 8).

\begin{tabular}{|c|c|c|c|}
\hline 〈grade〉 & 〈diplômé〉 & 〈curriculum〉 & 〈personne inscrite au curriculum〉 \\
\hline bachelier, -ière & bachelier, -ière & $\begin{array}{l}\text { bac } \\
\text { [calauréat] }\end{array}$ & $\begin{array}{l}\text { étudiant-e en ixième } \\
\text { année de bac[calauréat] }\end{array}$ \\
\hline maître* & maître ${ }^{\star \star}$ & maîtrise ${ }^{\star \star \star}$ & $\begin{array}{l}\text { étudiant-e en ixième } \\
\text { année de maîtrise }\end{array}$ \\
\hline docteur-e & docteur-e & doctorat & doctorant-e \\
\hline
\end{tabular}

* Ou masteur-e si l'on privilégie l'emprunt phonétiquement adapté à l'emprunt interne par remotivation du terme existant maître.

** Ou masteur-e (idem).

$\star \star \star$ Ou masteur (idem).

Tableau 7 : Scénario théorique de terminologie normative

23. Non-respect du décret de féminisation des noms de grades, fonctions et métiers; usage hasardeux de la majuscule à l'initiale des termes; flous notionnels; coexistence de dénominations concurrentes héritées de l'ancienne nomenclature dans certaines sources juridiques. 


\begin{tabular}{|c|c|c|c|}
\hline 〈grade〉 & 〈diplômé〉 & 〈curriculum〉 & $\begin{array}{l}\text { 〈personne inscrite } \\
\text { au curriculum〉 }\end{array}$ \\
\hline bachelier, -ière & bachelier, -ière & bac [calauréat] & $\begin{array}{l}\text { étudiant-e en ixième } \\
\text { année de bac[calauréat] }\end{array}$ \\
\hline master & $\begin{array}{l}\text { titulaire d'un grade } \\
\text { de master }\end{array}$ & master & $\begin{array}{l}\text { étudiant-e en ixième } \\
\text { année de master }\end{array}$ \\
\hline docteur-e & docteur-e & doctorat & doctorant-e \\
\hline
\end{tabular}

Tableau 8 : Proposition réaliste de terminologie aménagée

Le SLF a relayé nos remarques et notre proposition d'aménagement réaliste auprès du Conseil de la langue française et de la politique linguistique de la FWB, lequel s'en est saisi et a rédigé en décembre 2010 un avis (consultatif) au ministre de l'Enseignement supérieur. Et une proposition de décret visant à modifier la terminologie de l'enseignement supérieur suivant le scénario de terminologie normative telle que présentée dans le tableau 7 a été déposée au Parlement le 29 novembre $2011^{24}$. Malgré tout, à ce jour, aucune décision politique n'a été prise. Ceci n'a certes pas empêché la mise en ligne des fiches terminologiques validées par le Conseil de la langue dans BelTerme, la banque de données terminologique du SLF de la FWB. Mais une telle situation est pour le moins ambigüe. La lourdeur administrative qu'impliquerait une révision du décret et les complications qui s'ensuivraient au sein des administrations et institutions supérieures suffisent-elles à expliquer le manque de réactivité de l'exécutif de l'enseignement supérieur en FWB? Nous tendons à penser que non, le décret de Bologne ayant, du reste, déjà fait l'objet de trois modifications publiées au Moniteur belge entre 2004 et 2006.

\section{Les enjeux d'un choix terminologique}

Le phénomène que nous avons décrit nous semble soulever diverses questions qui correspondent à autant d'enjeux de société aux yeux du linguiste.

\section{Langage juridique vs langue de référence}

Le premier est celui du choc entre le langage juridique et la langue de référence. Dans un texte de loi, le juriste tente de construire un monde idéal où tout est prévu. Pour y parvenir, il «définit en droit», restreignant ou contraignant le sens d’un lexème de la langue courante ou spécialisée. Cette démarche est

24. Parlement de la Fédération Wallonie-Bruxelles, Proposition de décret - 285 (2011-2012), en ligne [http://www.pfwb.be/le-travail-du-parlement/doc-et-pub/documents-parlementaireset-decrets/dossiers/001357946] (dernière consultation : 19 février 2013). 
onomasiologique dès lors qu'elle revient à choisir un signifiant pour désigner le concept juridique que l'on entend créer. S'agissant de construire un enseignement supérieur, le législateur commencera son texte par des définitions contraignantes, comme :

Article 6. - § 1. Pour l'application du présent décret, et de ses arrêtés d'exécution, l'on entend par:

[...]

Bachelier : grade académique sanctionnant des études de premier cycle de 180 crédits au moins. (Moniteur belge, 2004, art. 6, § 1)

Tous les textes de la même "galaxie juridique » se réfèreront à cette définition, qui sera modifiée ultérieurement si nécessaire, par exemple pour changer le nombre de crédits correspondant à un premier cycle universitaire dans le cadre d'une nouvelle harmonisation européenne.

Le choix du terme relève largement de l'arbitraire et la nécessité même de définir les choses sous un angle juridique implique que l'on ne se soucie guère du choc qu'il pourrait engendrer avec d'autres usages. Mais dès que les mots prennent une acception particulière dans le domaine juridique, ils sont susceptibles de poser des problèmes de compréhension à l'usager. Être une personne handicapée se définit en droit à travers des conditions qui ne relèvent pas du bon sens commun ${ }^{25}$. Les difficultés de compréhension des lois et règlements que rencontre le citoyen relèvent largement de cette problématique et mériteraient assurément une très large étude associant lexicologues, sociolinguistes et juristes.

Le cas du terme bachelier tient presque de la caricature, tant il est évident, à nos yeux, que son choix pour désigner un curriculum relève d'une méconnaissance des structures de la langue. On est en droit de penser qu'un locuteur francophone de naissance sera interpellé par un énoncé du type «s'inscrire en bachelier ou en master ». Au-delà de ce cas d'école, il est regrettable que l'absence de réelle politique linguistique conduise trop souvent des pouvoirs exécutifs à créer des termes porteurs d'ambigüité ou dont la forme morphosyntaxique est contraire aux règles de la langue française.

\section{Statalisme et francophonie}

Les législateurs de chaque pays ont disposé d'une entière liberté pour mettre en œuvre le processus de Bologne, qui a vu le jour dans le cadre du Conseil de l'Europe. On n'est donc pas dans le cas de figure d'un texte normatif européen ou international qui s'applique tel quel dans l'ordre juridique national. Il ne

25. Voir, par exemple, l'article premier de l'Arrêté royal du 6 octobre 2005 portant diverses mesures en matière de sélection comparative de recrutement et en matière de stage. 
s'agit pas davantage d'un cas de transcription du droit communautaire dont la phraséologie et la terminologie sont directement inspirées par le travail des rédacteurs et des traducteurs de l'Union européenne.

Aucune concertation linguistique n'étant prévue entre les pays européens de langue française, chaque pays ou entité fédérée francophone a agi en totale liberté pour créer ses statalismes, c'est-à-dire des faits de langue observés essentiellement à l'intérieur des frontières d'un seul État (Pohl, 1984) :

- Confédération helvétique : bachelor - master - doctorat (Conférence universitaire suisse, 2008, art. 1);

- Fédération Wallonie-Bruxelles : bachelor - master-doctorat (Moniteur belge, 2004, art. 6, par. 1);

- Grand-Duché de Luxembourg : bachelor - master - doctorat (ministère de l'Éducation nationale, 2011, chap. 5, art. 6);

- République française : licence - master - doctorat Vournal officiel de la République française, 2002, art. 3).

Le nœud du problème réside, vraisemblablement, dans l'utilisation du terme baccalauréat en français de France, vécu comme français de référence. Il est très vraisemblable que les différents législateurs aient voulu éviter un mot désignant des études secondaires en France, les chances étant minimes de voir la République française accepter de changer le niveau d'un diplôme ${ }^{26}$ qui, dans l'imaginaire collectif francophone, est lourdement connoté. Seule une étude historique du mouvement qui a conduit à des prises de décision terminologiques au sein de chaque État permettrait de corroborer cette hypothèse. La vérité linguistique demeure, toutefois, que le terme baccalauréat est l'équivalent de bachelor au Québec et qu'il a été utilisé en Belgique par certaines universités jusqu’à la fin du $20^{\mathrm{e}}$ siècle pour désigner des diplômes complémentaires de premier cycle.

La vitalité des emprunts dans les pays multilingues a fait l'objet de nombreuses descriptions (voir notamment Latin, Poirier, 2000). La perméabilité des francophones de Belgique à la langue anglaise (Dierickx, 1997) explique certainement un recours à une matrice externe. Le choix du terme bachelier pour rendre bachelor, calque abusif, relève au mieux d'un pis-aller. Il constitue à nos yeux une manifestation de l'insécurité linguistique du locuteur francophone de Belgique, fût-il juriste et unilingue, baignant dans une diglossie plus ou moins assumée (Francard, 1997, p. 235 et Moreau, 1997).

Le paradoxe de la situation est de constater qu'à défaut de disposer d'un organe multilatéral de gestion de la langue commune, une terminologie ad hoc aurait pu être adoptée si le processus de Bologne avait débouché sur un texte de droit international prenant rang, tel quel, dans les législations nationales. Dans ce cas de figure, la ratification reconnait le travail du traducteur.

26. Les francophones de l'étranger ignorent généralement que le baccalauréat français est un premier titre universitaire. 


\section{Statalisme et statut du terme}

Les statalismes créés se distinguent toutefois par un aspect nouveau : le "pensé » est commun à plusieurs États. L'harmonisation des systèmes d'enseignement supérieur est un processus de normalisation d'un univers extralinguistique. Les théories wüsteriennes d'aménagement terminologique (Wüster, 1979) s'y appliquent à merveille, les «mots pour le dire " n'étant que de simples étiquettes collées sur des concepts dont les contours ont fait l'objet d'une négociation internationale. Le choc frontal avec l'usage peut alors s'expliquer par le fait que les termes sont susceptibles de perdre leur dimension de signes linguistiques, le signifié n'entretenant plus un lien indéfectible avec le signifiant et se confondant avec un concept extérieur à la langue.

La démonstration en est assurément apportée par la décision du législateur, qui considère que l'on devra "s'inscrire en bachelier», comme s'il était possible de «s'inscrire en bâtonnier» (pour accéder un jour au bâtonnat), «en aérostier» (pour se former à l'aérostat) ou encore «en auditeur» (pour pouvoir pratiquer l'auditorat)...

Le choix d'un terme ressenti comme contraire à l'habitude linguistique débouche inévitablement sur une forme de diglossie entre la langue juridicoadministrative et la langue informelle, par laquelle le locuteur se réinvente un usage. Quitte à le faire dans un sentiment d'insécurité linguistique manifeste.

\section{Références}

DIERICKX Jean, 1997, "Le français et l'anglais», Le français en Belgique. Une langue, une communauté, D. Blampain, A. Goosse, J.-M. Klinkenberg, M. Wilmet éd., Louvain-la-Neuve, Duculot et Communauté française de Belgique, p. 307-318.

FRANCARD Michel, 1997, "Le français en Wallonie», Le français en Belgique. Une langue, une communauté, D. Blampain, A. Goosse, J.-M. Klinkenberg, M. Wilmet éd., Louvain-la-Neuve, Duculot et Communauté française de Belgique, p. 229-237.

LATIN Danièle, Poirier Claude éd., 2000, Contacts de langues et identités culturelles. Perspectives lexicographiques, Montréal, Agence universitaire de la francophonie Québec, Presses de l'université Laval.

MoREAu Marie-Louise, 1997, «Le bon français de Belgique. D’un divorce entre norme et discours sur la norme», Le français en Belgique. Une langue, une communauté, D. Blampain, A. Goosse, J.-M. Klinkenberg, M. Wilmet éd., Louvain-la-Neuve, Duculot et Communauté française de Belgique, p. 391-399.

РонL Jacques, 1984, "Le statalisme», Travaux de linguistique et de littérature, t. XXII, $\mathrm{n}^{0} 1$, p. 251-264.

VAN CAMPENhoudT Marc, 2000, «De la lexicographie spécialisée à la terminographie. 
Vers un "métadictionnaire” ? ", Le sens en terminologie, Ph. Thoiron, H. Béjoint éd., Lyon, Presses universitaires de Lyon (Travaux du CRTT), p. 127-152.

- 2001, "Pour une approche sémantique du terme et de ses équivalents », International Journal of Lexicography, septembre 2001, vol. XIV, n³ 3, p. 181-209.

WüSTER Eugen, 1979, Einführung in die allgemeine Terminologielehre und terminologische Lexikographie, 2 vol., New York, Spring.

\section{Textes officiels}

Directives pour le renouvellement coordonné de l'enseignement des hautes écoles universitaires suisses dans le cadre du processus de Bologne (Directives de Bologne) du 4 décembre 2003, $3^{\mathrm{e}}$ édition, Berne, Conférence universitaire suisse [www.cus.ch/ wFranzoesisch/publikationen/richtlinien/BOL-RL-2008-Fr-V2.pdf] (dernière consultation le 27 mars 2013).

Code de l'Éducation nationale, législation jusqu'au 31 décembre 2011, Luxembourg, ministère de l'Éducation nationale, de la Formation professionnelle et des Sports [www.legilux.public.lu/leg/textescoordonnes/compilation/code_education_nationale/CHAPITRE_5.pdf] (dernière consultation le 27 mars 2013).

Décret nº 2002-481 du 8 avril 2002 relatif aux grades et titres universitaires et aux diplômes nationaux, Journal officiel de la République française, 10 avril 2002, n84, p.6324 [http://www.legifrance.gouv.fr/affichTexte.do?cidTexte=JORFTEXToooo00585864] (dernière consultation le 27 mars 2013).

Décret définissant l'enseignement supérieur, favorisant son intégration dans l'espace européen de l'enseignement supérieur et refinançant les universités, Moniteur belge, 18 juin 2004, p.45239-45301, mises à jour en ligne [www.gallilex.cfwb.be/fr/leg res_01.php?ncda=28769 \& referant $=$ l01] (dernière consultation le 27 mars 2013). 